\title{
All-optical quantum simulator of qubit noisy channels
}

\author{
Simone Cialdi, ${ }^{1, a), b)}$ Matteo A. C. Rossi, ${ }^{1, b)}$ Claudia Benedetti, ${ }^{1, b)}$ Bassano Vacchini, ${ }^{1,2}$ \\ Dario Tamascelli, ${ }^{1, b)}$ Stefano Olivares, ${ }^{1, b)}$ and Matteo G. A. Paris ${ }^{1, b)}$ \\ ${ }^{1}$ Quantum Technology Lab, Dipartimento di Fisica, Università degli Studi di Milano, I-20133 Milano, Italy \\ ${ }^{2}$ INFN, Sezione di Milano, I-20133 Milano, Italy
}

(Received 20 December 2016; accepted 4 February 2017; published online 23 February 2017)

\begin{abstract}
We suggest and demonstrate an all-optical quantum simulator for single-qubit noisy channels originating from the interaction with a fluctuating field. The simulator employs the polarization degree of freedom of a single photon and exploits its spectral components to average over the realizations of the stochastic dynamics. As a proof of principle, we run simulations of dephasing channels driven either by Gaussian (Ornstein-Uhlenbeck) or non-Gaussian (random telegraph) stochastic processes. Published by AIP Publishing. [http://dx.doi.org/10.1063/1.4977023]
\end{abstract}

A quantum simulator (QS) is a quantum system where the initial preparation and the subsequent time evolution may be controlled and monitored. QSs may be exploited to mimic the dynamics of other quantum systems that are less accessible or less controllable. ${ }^{1}$ The inherent parallel structure of QSs makes them suitable to solve problems that are intractable on conventional supercomputers, e.g., the simulation of the dynamics of a many-particle system. In particular, photonic quantum simulators may be used at room temperature, thanks to the fact that photons do not interact with each other. ${ }^{2-5}$ Moreover, photons may propagate in free space or in waveguides and thus may be used to simulate complex structures with a long range interaction.

In this Letter, we suggest and demonstrate an all-optical QS that exploits the spectral components of a single-photon state to perform the parallel sum of about one hundred complex numbers. In order to demonstrate the operation of our QS, we run the simulation of two different single-qubit dephasing channels, arising from the interaction of the quantum system with an external fluctuating (stochastic) field. These channels correspond to exact effective models for the interaction of qubits with complex environments ${ }^{6}$ and are found in a variety of physical implementations such as solidstate, superconducting qubits, and magnetic systems. In turn, these systems are crucial in the quest for quantum technologies and have been extensively studied. ${ }^{7-9}$ Upon exploiting our QS, the interaction with any fluctuating field may be simulated and analyzed. In particular, here we focus on two paradigmatic channels, driven either by (Gaussian) OrnsteinUhlenbeck (OU) stochastic noise $\mathrm{e}^{10,11}$ or by (non-Gaussian) random telegraph noise (RTN). ${ }^{12,13}$

For a system interacting with a fluctuating field, the density operator $\rho(t)$ describing the state of the system at any time $t$ corresponds to the average over all possible realizations of the stochastic process. The implementation of such a state in the lab would therefore require the simultaneous generation of a large number of stochastic trajectories of the process. Here, we show that this procedure may be avoided and that the average over the realizations of the noise may be

\footnotetext{
${ }^{\text {a)} E l e c t r o n i c ~ m a i l: ~ s i m o n e . c i a l d i @ u n i m i . i t ~}$

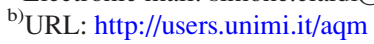

obtained in parallel. The quantum information carrier is a photon. The polarization of the photon is used to encode the state of a qubit, whereas its spectral components are exploited to implement the trajectories of the stochastic process describing the fluctuating field.

We simulate the evolution of a single qubit evolving under a time-dependent Hamiltonian of the form $H(t)=H_{0}$ $+H_{\text {int }}=\varepsilon \sigma_{z}+X(t) \sigma_{z}$, where $\sigma_{z}$ is the Pauli matrix and $\varepsilon$ determines the energy splitting of the qubit. $H_{\text {int }}$ describes the interaction of the system with a fluctuating environment and $X(t)$ is an arbitrary real-valued continuous-time stochastic process. The environment induces decoherence but does not exchange energy with the system. If the qubit is initially prepared in the state $\left|\psi_{0}\right\rangle=(1 / \sqrt{2})(|0\rangle+|1\rangle)$, the evolved state is given by $\rho(t)=\left\langle U(t) \rho_{0} U^{\dagger}(t)\right\rangle$, where we introduced $\rho_{0}=\left|\psi_{0}\right\rangle\left\langle\psi_{0}\right|, U(t)=\exp \left[-i \int_{0}^{t} H(\tau) d \tau\right]$ is the evolution operator, and $\langle\cdot\rangle$ denotes the expectation value over the realizations of the stochastic process, i.e., of the noise. In the interaction picture, we have

$$
\rho(t)=\frac{1}{2}\left(\begin{array}{cc}
1 & \left\langle e^{-2 i \Phi(t)}\right\rangle \\
\left\langle e^{2 i \Phi(t)}\right\rangle & 1
\end{array}\right),
$$

where $\Phi(t)=\int_{0}^{t} X(\tau) d \tau$. In order to obtain the state of the system at any time $t$, we should compute the average of a sufficiently large collection of independent realizations (sample-paths) of the stochastic process $X(t)$. Each samplepath is a real scalar function $\Phi_{r}(t)=\int_{0}^{t} X_{r}(\tau) d \tau$ that corresponds to the phase shift induced by a particular realization $X_{r}(\tau)$, with $r$ running on the sample index.

In the following, we describe an experimental all-optical setup that allows us to obtain the evolved state upon the generation of $n$ sample-paths in a single run. In particular, the qubit (polarization) state at time $\bar{t}$ will be given by $\rho(\bar{t})$ $=\frac{1}{n} \sum_{r=1}^{n}\left|\psi_{r}(\bar{t})\right\rangle\left\langle\psi_{r}(\bar{t})\right|$, where, according to Eq. (1), $\left|\psi_{r}(\bar{t})\right\rangle$ $=(1 / \sqrt{2})\left(e^{-2 i \Phi_{r}(\bar{t})}|H\rangle+|V\rangle\right)$. In Fig. 1, we show a schematic diagram of the experimental apparatus. The frequency-entangled two-photon state is generated by parametric down-conversion (PDC) with a diode pump laser @ $405.5 \mathrm{~nm}$ by using a beta-barium borate (BBO) crystal ( $1 \mathrm{~mm}$ thick). The laser is temperature stabilized and generates $40 \mathrm{~mW} @ 70 \mathrm{~mA}$. The two photons are then collected 


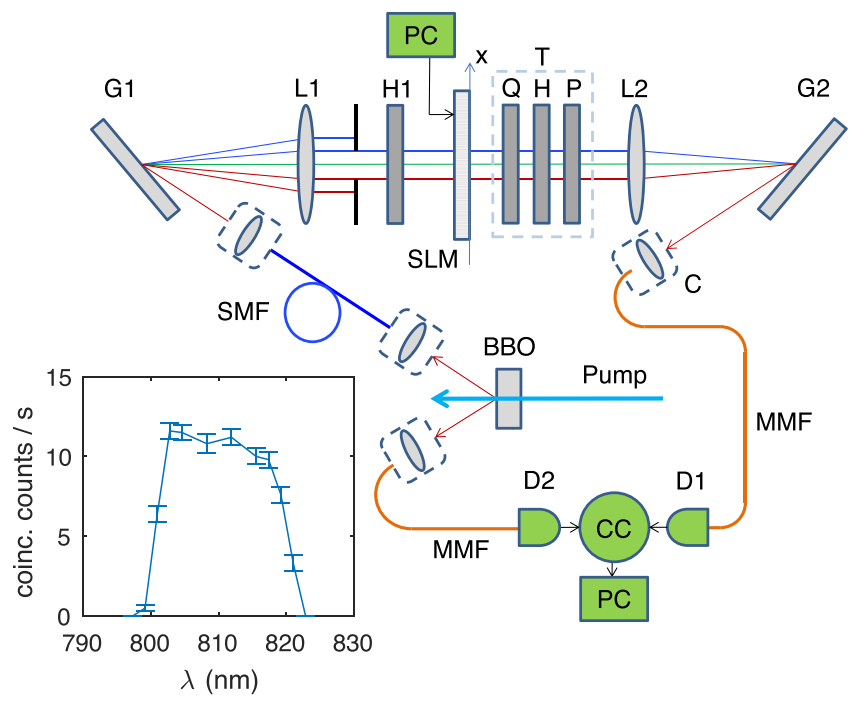

FIG. 1. Schematic diagram of our setup. Pump, $405.5 \mathrm{~nm}$ laser diode; BBO, Beta barium borate nonlinear crystal; SMF, single-spatial-mode and polarization preserving fiber; MMF, multimode fiber; G1-G2, gratings; L1-L2, lens; H1, half-wave-plate; SLM, spatial light modulator; T, tomographic apparatus; C, optical coupler; D1-D2, single photon detectors; and CC, coincidences counter. The inset shows the measured PDC spectrum.

by two fiber couplers and sent into a single-spatial-mode and polarization-preserving fiber (SMF) and a multimode fiber (MMF), respectively. When the idler photon enters the coupler, it travels entirely through the fiber (MMF) towards the single photon detector (D2). Conversely, the signal photon, after a short fiber (SMF), enters a $4 \mathrm{~F}$ system, ${ }^{14}$ i.e., propagates in the air, through few optical devices [the gratings G1 and G2 (1714 lines $/ \mathrm{mm})$ and lens L1 and $\mathrm{L} 2(f=500 \mathrm{~mm})]$ an half-wave plate (H1), that we use for the input state preparation, a spatial light modulator (SLM), and a tomographic apparatus $(\mathrm{T})^{15,16}$ to reconstruct the output state. At the end of the $4 \mathrm{~F}$ system, the signal photon is coupled to a multimode fiber and reaches the single photon detector (D1). Finally, an electronic device measures the coincidence counts (CC) and sends them to the computer (PC). The tomographic apparatus $(\mathrm{T})$ is composed of a quarter-wave plate $(\mathrm{Q})$, an half-wave plate $(\mathrm{H})$, and a polarizer $(\mathrm{P})$. The SLM is a 1D liquid crystal mask (640 pixels, $100 \mu \mathrm{m} /$ pixel) and is placed on the Fourier plane between the two lenses L1 and L2 of the 4F system (see Fig. 1). The SLM is controlled by the computer (PC) and is used to introduce a different phase $\Phi_{r}(\vec{t})$ for each pixel. In the Fourier plane, the spectral components of the signal photon are linearly dispersed $(1.82 \mathrm{~nm} / \mathrm{mm})$.

In order to measure the PDC spectra, we used a $2 \mathrm{~mm}$ slit on the Fourier plane of the 4F system. We calibrated the slit using a graduated reference on the Fourier plane, and for each slit position (and therefore for each wavelength), we recorded coincidence counts from the detectors. In the inset of Fig. 1, we show the measured PDC spectrum. We observe that it is selected by the limited width of the $\mathrm{H} 1$ plate mount, in such a way that the intensity of the spectral components impinging on the SLM is almost constant, a relevant feature to implement our QS. For this reason, we are limited to use $n=100$ out of the 640 pixel available on the SLM.

When leaving the BBO, signal $(s)$ and idler $(i)$ photons are in the pure state $\int d \omega f(\omega)|H\rangle_{s} \otimes|\omega\rangle_{s} \otimes|H\rangle_{i} \otimes|-\omega\rangle_{i}{ }^{17}$ where $H$ denotes the horizontal polarization and $\omega$ is the spectral shift with respect to the PDC central component $\omega_{0}=\omega_{p} / 2$, where $\omega_{p}$ is the pump laser frequency. We point out that the polarization and frequency degrees of freedom of the two photons are independent of each other, and thus, upon the detection of an idler photon, the conditional state of the signal photon, i.e., the partial trace over the idler degrees of freedom, is given by the mixed state

$$
\rho_{S E}=\rho_{S} \otimes \rho_{E}=|H\rangle\left\langle\left. H\left|\otimes \int d \omega\right| f(\omega)\right|^{2} \mid \omega\right\rangle\langle\omega| .
$$

The initial system-environment state is therefore factorized, and this warrants the existence of the reduced dynamics. ${ }^{18}$ The polarization of the idler photon encodes a qubit, while the spectral/spatial degrees of freedom may be considered as the environment. The grating G1 (see Fig. 1) disperses linearly the photon spectral components $\omega$, and the lens L1 focuses them on the Fourier plane of the 4F system where the SLM is placed. Each spectral component $\omega$ is characterized by a Gaussian spatial profile ( $60 \mu \mathrm{m}$ FWHM) centered in the spatial coordinate $x$. We have $\omega=\alpha x$, where $\alpha=1.82 \mathrm{~nm} / \mathrm{mm}$. In order to emphasize that the spectral components are spatially dispersed, we use the notation $|x\rangle=|\omega(x)\rangle$. The half-wave plate H1 rotates the polarization of the signal photon, turning the initial state of the system to $\left|\psi_{S}(0)\right\rangle=(1 / \sqrt{2})(|H\rangle+|V\rangle)$. Introducing with the notation $\left|\eta_{r}\right\rangle$ the $r$-th pixel of the SLM, we have $|x\rangle=\sum_{r} \eta_{r}(x)\left|\eta_{r}\right\rangle$, where $\left|\eta_{r}(x)\right|^{2}$ is the probability that the component $x$ passes through the $r$-th pixel. In this notation, the identity $\mathbb{1}=\sum_{r}\left|\eta_{r}\right\rangle\left\langle\eta_{r}\right|$ expresses the fact that all detectable components pass through the pixels.

The initial state of the environment then reads $\rho_{E}=\sum_{r, s} A_{r s}\left|\eta_{r}\right\rangle\left\langle\eta_{s}\right|$, where

$$
A_{r s}=\int d x|f(x)|^{2} \eta_{r}(x) \eta_{s}^{*}(x) .
$$

The matrix $A_{r s}$ is positive definite with trace equal to one. The SLM imprints a pixel-dependent phase on the horizontal polarization component, which we denote by $e^{-2 i \Phi_{r}(\bar{t})}$ (see Eq. (1)). The unitary interaction operator can therefore be written in the form

$$
U(\bar{t})=\exp \left[-2 i P_{H} \otimes \sum_{r} \Phi_{r}(\bar{t}) P_{r}\right],
$$

where $P_{H}=|H\rangle\langle H|$ and $P_{r}=\left|\eta_{r}\right\rangle\left\langle\eta_{r}\right|$. As a result, $U(\bar{t})|H\rangle$ $\otimes\left|\eta_{r}\right\rangle=e^{-2 i \Phi_{r}(\bar{t})}|H\rangle \otimes\left|\eta_{r}\right\rangle$, while vertically polarized states are left unchanged. Taking the marginal of $\rho_{S E}(\bar{t})=U(\bar{t})$ $\left(\rho_{S}(0) \otimes \rho_{E}\right) U(\bar{t})^{\dagger}$, we thus obtain

$$
\rho_{S}(\bar{t})=\frac{1}{2} \sum_{r} A_{r r}\left(\begin{array}{cc}
1 & \mathrm{e}^{-2 i \Phi_{r}(\bar{t})} \\
\mathrm{e}^{2 i \Phi_{r}(\bar{t})} & 1
\end{array}\right),
$$

so that the only matrix element affected by the dynamics is $\left\langle H\left|\rho_{S}(\bar{t})\right| V\right\rangle$. In our case for the diagonal elements, we have $A_{r r}=1 / n(n=100)$ because the selected PDC spectrum is basically rectangular. However, due to the imperfections of the experimental apparatus, in each realization, the state is 
not exactly pure but rather of the form $\rho_{S \text {, exp }}=p \rho_{S}$ $+(1-p) \rho_{\text {mix }}$, where $\rho_{\text {mix }}=\frac{1}{2}|H\rangle\left\langle H\left|+\frac{1}{2}\right| V\right\rangle\langle V|$ is the maximally mixed state, so that the relevant quantity to be measured is

$$
\left\langle H\left|\rho_{S, \exp }(\bar{t})\right| V\right\rangle=\frac{1}{2} p\left\langle e^{-2 i \Phi_{r}(\bar{t})}\right\rangle_{n} .
$$

In our setup, the average over the realizations of the noise is performed by (coherently) collecting the different spatial components $|\omega\rangle$ through the lens L2 and the grating G2 into a multimode fiber. The state reconstruction is performed by the tomographic apparatus T placed between the SLM and the lens L2.

In the following, we show the results obtained by running simulations of two dephasing channels driven either by Ornstein-Uhlenbeck Gaussian noise or non-Gaussian random telegraph noise. Apart from providing a convenient description of many realistic environments, dephasing channels also permit a simple assessment of the non-Markovian character of the reduced dynamics of the system. ${ }^{19}$ This criterion relies on the study of the behaviour in time of the distinguishability among different initial states of the system evolved according to the same reduced dynamics. The distinguishability between states is quantified by their trace distance defined as $D(t)=\frac{1}{2}\left\|\rho_{1}(t)-\rho_{2}(t)\right\|_{1}$, that is half the trace norm of the difference of the two statistical operators. Non-Markovianity is associated with revivals in time of this quantity. In particular, it can be shown ${ }^{20}$ that, for a dephasing map, the highest sensitivity to these revivals is obtained by looking at the modulus of the coherences of the statistical operator $\rho(t)$ of Eq. (1), which indeed equals the trace distance among the pair of states better witnessing non-Markovianity.

For the RTN, the realization $X_{r}(\bar{t})$ flips randomly between the values \pm 1 with a switching rate $\gamma$. In our case for each step of the realization, the simulation time $\bar{t}$ is incremented by $\delta \bar{t}=0.001$ in units of $1 / \gamma$. The flip probability at each step is given by $\delta P=1-e^{-\gamma \delta \bar{t}}$. The initial values $X_{r}(0)$ are selected randomly with equal probability between \pm 1 for each pixel. In the case of the OU process, we have

$$
X_{r}(\bar{t}+\delta \bar{t})=(1-2 \gamma \delta \bar{t}) X_{r}(\bar{t})+2 \sqrt{\gamma} d W(\bar{t}),
$$

where $d W(\bar{t})$ is a Wiener increment with the mean equal to zero and standard deviation $\sigma=\sqrt{\delta \bar{t}}$. For each realization (i.e., for each pixel), we impose the initial condition $X_{r}(0)=0$. Both models are analytically solvable, ${ }^{13,21}$ and it is known that any dephasing map induced by a Gaussian stochastic process is Markovian, while RTN gives a nonMarkovian map for $\gamma<2 .{ }^{13}$ In Fig. 2(a) and Fig. 2(b), we plot the experimental results in the case of the RTN and OU process, respectively. In both cases, we have $\gamma=0.1$ in arbitrary units. We note the presence of strong revivals in the RTN case, according to the non-Markovian character of the dynamics. In the OU case, the off-diagonal element of $\rho_{S}(\bar{t})$ decays monotonically, as expected for a Markovian dynamics. For each point of the graph $\left(\bar{t}_{i}=i \times 50 \delta \bar{t}\right)$, we send to the pixels the phases $\Phi_{r}\left(\bar{t}_{i}\right)=\int_{0}^{\bar{t}_{i}} X_{r}(\tau) d \tau$, and we reconstruct the state with the tomographic method by performing four projective measurements. ${ }^{15,16,22}$ We use an acquisition time
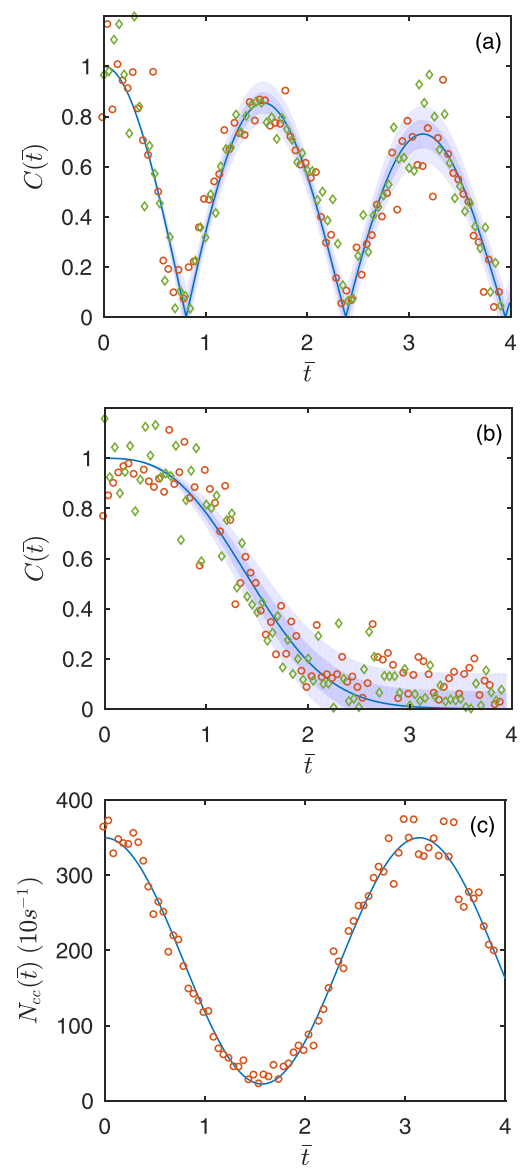

FIG. 2. (a) and (b) Dynamics of the off-diagonal element of $\rho_{S}(\bar{t}), C(\bar{t})$ $=\left|\left\langle e^{-2 i \Phi_{r}(t)}\right\rangle_{n}\right|$, for RTN (a) and OU (b) with $\gamma=0.1$. Red circles and green diamonds represent the data obtained, respectively, with tomographic reconstruction of $\rho_{S, \exp }(\bar{t})$ and projection onto the state $|+\rangle$. The blue line is the analytic solution of the model. The shades represent intervals of $1 \sigma$ (darker) and $2 \sigma$ (lighter) around the analytic solution, where $\sigma$ is the standard deviation of paths obtained with 100 realizations of the stochastic process. Note that the noise for small $\bar{t}$ is due to the Poissonian fluctuations on the coincidence counts. (c) Coincidence counts $N_{\mathrm{cc}}(\bar{t})$ in the case of RTN with $\gamma=0$, and the blue line is the fit with the function $N_{\mathrm{cc}}=N(1+p \cos (2 \vec{t}))$.

of $10 \mathrm{~s}$ for each measure of coincidence counts. For a pure dephasing dynamics, one has

$$
D(t)=\left|\left\langle e^{-2 i \Phi(t)}\right\rangle\right| \approx\left|\left\langle e^{-2 i \Phi_{r}(\bar{t})}\right\rangle_{n}\right| \equiv C(\bar{t}) .
$$

Notice that in order to obtain the non-Markovianity from the revivals of the trace distance, we need the factor $\frac{1}{2} p$. Indeed, while the trace distance is in principle bounded by one, here we estimate its value from the reduced dynamics of the offdiagonal matrix elements, whose actual value depends on the purity of the system state. The latter is known only in average, and it is also affected by experimental uncertainty due to the Poissonian statistics of photon counting. The quantity $C(\bar{t})$ is shown in Fig. 2(a) and Fig. 2(b) as a function of $\bar{t}$ for RTN and OU noise, both with $\gamma=0.1$. Notice that $\left\langle e^{-2 i \Phi(\bar{t})}\right\rangle$ is real-valued because the two considered stochastic processes have zero mean (and indeed, from the tomographic measures, we find that the imaginary part of $\left\langle e^{-2 i \Phi_{r}(\bar{t})}\right\rangle_{n}$ is zero within the experimental uncertainty). Thus, in order to estimate the trace distance, we can perform just one projective measure on the state $|+\rangle=(1 / \sqrt{2})(|H\rangle+|V\rangle)$ since we have $\left\langle+\left|\rho_{S, \exp }\right|+\right\rangle=\frac{1}{2}\left(1+p \operatorname{Re}\left\langle e^{-2 i \Phi_{r}(\bar{t})}\right\rangle_{n}\right)$. In order to 

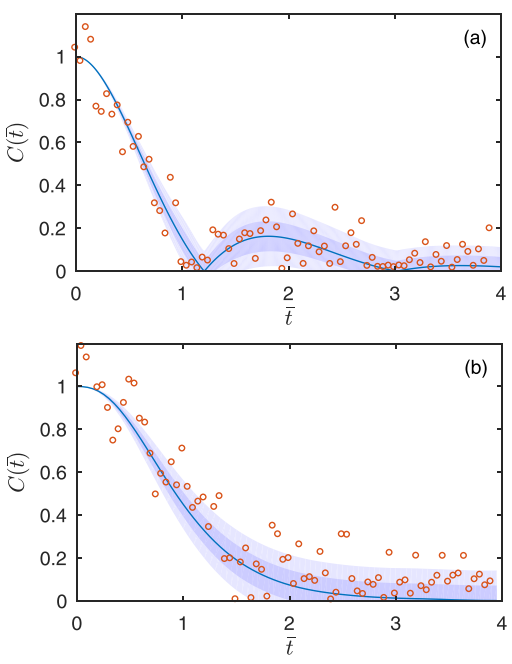

FIG. 3. Dynamics of the off-diagonal element of $\rho_{S}(\bar{t}), C(\bar{t})=\left|\left\langle e^{-2 i \Phi_{r}(\bar{t})}\right\rangle_{n}\right|$, vs $\bar{t}$ evaluated by the method of the projection onto the state $|+\rangle$ in the case $\gamma=1$ for RTN (a) and OU (b) stochastic process. The blue line is the analytic solution and the blue shades represent intervals of $1 \sigma$ (darker) and $2 \sigma$ (lighter) around the analytical solution, where $\sigma$ is the standard deviation of paths obtained with 100 realizations of the stochastic process.

obtain the parameter $p$, we acquire a reference measure using the RTN with $\gamma=0$ (i.e., static noise). In this case, we have $\left\langle e^{-2 i \Phi_{r}(\bar{t})}\right\rangle=\cos (2 \bar{t})$. In Fig. 2(c), we can see the coincidence counts vs. the simulation time $\bar{t}$ in the case of the RTN with $\gamma=0$. From the fit (blue solid line) with the function $N_{\mathrm{cc}}(\vec{t})=N(1+p \cos (2 \vec{t}))$, we find $p=0.88 \pm 0.02$ and $N=186 \pm 2$. Thus, in the general case, we can write as follows: $\left\langle e^{-2 i \Phi_{r}(\bar{t})}\right\rangle_{n}=\left(N_{\mathrm{cc}}(\bar{t})-N\right) / p$. In Figs. 2(a) and 2(b), we can also see the comparison between the tomographic method (red circles) and the method based on the projection on the state $|+\rangle$ (green diamonds) in the case of the RTN and of the OU. We note that the two methods indeed give compatible results. In Fig. 3, we can see the results obtained by the projection method on the state $|+\rangle$ and with $\gamma=1$, for both RTN (a) and OU process (b). Note the decrease in nonMarkovianity of the RTN dynamics compared to the case with $\gamma=0.1$. In turn, the non-Markovianity vanishes when $\gamma \geq 2 .{ }^{13}$ In the case of the OU process, the dynamics remains Markovian as expected.

In conclusion, we have suggested and demonstrated an all-optical quantum simulator for single-qubit noisy channels. The simulated qubit is encoded in the polarization degree of freedom of a single-photon generated by parametric downconversion, whereas several realizations of the noise are achieved in a single shot by using a programmable spatial light modulator on the different spectral components of the photon.

As a proof of principle, we have run simulations of dephasing channels driven either by Gaussian (OrnsteinUhlenbeck) or non-Gaussian (random telegraph) stochastic processes. Upon increasing the number of pixels in the spatial light modulator, one may increase the number of realizations and perform more accurate simulations of noisy channels and complex classical environments.

This work was supported by EU through the collaborative project QuProCS (Grant Agreement No. 641277) and by UniMI through the H2020 Transition Grant.

${ }^{1}$ A. Aspuru-Guzik and P. Walther, Nature Phys. 8, 285 (2012).

${ }^{2}$ M. A. Broome, A. Fedrizzi, B. P. Lanyon, I. Kassal, A. Aspuru-Guzik, and A. G. White, Phys. Rev. Lett. 104, 153602 (2010).

${ }^{3}$ B. P. Lanyon, J. D. Whitfield, G. G. Gillett, M. E. Goggin, M. P. A. Almeida, I. Kassal, J. D. Biamonte, M. Mohseni, B. J. Powell, M. Barbieri, A. Aspuru-Guzik, and A. G. White, Nature Chem. 2, 106 (2010).

${ }^{4}$ X.-S. Ma, B. Dakic, W. Naylor, A. Zeilinger, and P. Walther, Nature Phys. 7, 399 (2011).

${ }^{5}$ X. Deng, S. Hao, H. Guo, C. Xie, and X. Su, Sci. Rep. 6, 22914 (2016).

${ }^{6}$ D. Crow and R. Joynt, Phys. Rev. A 89, 042123 (2014).

${ }^{7}$ J.-S. Xu, K. Sun, C.-F. Li, X.-Y. Xu, G.-C. Guo, E. Andersson, R. Lo Franco, and G. Compagno, Nat. Commun. 4, 2851 (2013); R. Lo Franco, B. Bellomo, E. Andersson, and G. Compagno, Phys. Rev. A 85, 032318 (2012).

${ }^{8}$ E. Paladino, M. Y. Galperin, G. Falci, and L. B. Altshuler, Rev. Mod. Phys. 86, 361 (2014).

${ }^{9}$ T. Yu and J. H. Eberly, Opt. Commun. 264, 393 (2006).

${ }^{10}$ C. Benedetti and M. G. A. Paris, Int. J. Quantum Inf. 12, 1461004 (2014).

${ }^{11}$ M. A. C. Rossi and M. G. A. Paris, J. Chem. Phys. 144, 024113 (2016).

${ }^{12}$ H. J. Wold, H. Brox, Y. M. Galperin, and J. Bergli, Phys. Rev. B 86, 205404 (2012).

${ }^{13}$ C. Benedetti, M. G. A. Paris, and S. Maniscalco, Phys. Rev. A 89, 012114 (2014).

${ }^{14}$ A. M. Weiner, Rev. of Sci. Instrum. 71, 1929 (2000).

${ }^{15}$ K. Banaszek, G. M. D’Ariano, M. G. A. Paris, and M. F. Sacchi, Phys. Rev. A 61, 010304 (1999).

${ }^{16}$ D. F. V. James, P. G. Kwiat, W. J. Munro, and A. G. White, Phys. Rev. A 64, 052312 (2001).

${ }^{17}$ A. Joobeur, B. E. A. Saleh, T. S. Larchuk, and M. C. Teich, Phys. Rev. A 53, 4360 (1996).

${ }^{18}$ P. Pechukas, Phys. Rev. Lett. 73, 1060 (1994).

${ }^{19}$ H.-P. Breuer, E.-M. Laine, and J. Piilo, Phys. Rev. Lett. 103, 210401 (2009).

${ }^{20}$ H.-P. Breuer, E.-M. Laine, J. Piilo, and B. Vacchini, Rev. Mod. Phys. 88, 021002 (2016).

${ }^{21}$ M. A. C. Rossi, C. Benedetti, and M. G. A. Paris, Int. J. Quantum Inf. 12, 1560003 (2014).

${ }^{22}$ S. Cialdi, A. Smirne, M. G. A. Paris, S. Olivares, and B. Vacchini, Phys. Rev. A 90, 050301(R) (2014). 